\title{
Efeitos da ingestão de diferentes soluções hidratantes nos níveis de hidratação e na frequência cardíaca durante um exercício de natação intervalado
}

\author{
Fabrícia G. Ferreira \\ Graciene L. de Almeida \\ João C. B. Marins
}

https://doi.org/10.5628/rpcd.07.03.319

\author{
Departamento de Educação Física \\ Laboratório de Performance H umana (LA PEH) \\ Universidade Federal de Viçosa \\ Brasil
}

\section{RESUMO}

Este estudo objectivou identificar os níveis de hidratação e a interferência na frequência cardíaca decorrente da adopção de diferentes procedimentos de hidratação durante um exercício de natação intervalado. Um total de 15 atletas do sexo masculi-

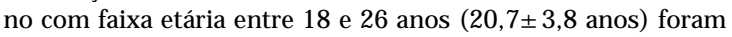
submetidos aos procedimentos: a) nenhum tipo de hidratação, b) hidratação com placebo c) hidratação com Gatorade ${ }^{\circledR}$. Cada tratamento experimental correspondeu a uma distância total de 4150 metros divididos em 250 metros de aquecimento; 1 × 400 metros à velocidade máxima; 1 x 100 metros em recuperação; $10 \times 250$ metros à 85-90\% da velocidade máxima para esta distância, com interval os de 50 metros de recuperação e 400 metros em velocidade máxima. Foi mensurada a frequência cardíaca, o peso corporal antes e depois de cada teste, a quantidade de líquido consumido durante o exercício e a urina produzida, para estabelecer os níveis de hidratação. O tratamento estatístico indicou não haver diferença estatisticamente significativa $(p>0,05)$ no efeito tempo e entre os grupos em nenhum dos dois parâmetros analisados. Pode-se concluir que os procedimentos de hidratação adoptados não influenciaram na resposta da frequência cardíaca e nível de hidratação durante o modelo experimental desenvolvido.

Palavras-chave: hidratação, desidratação, natação e freqüência cardíaca

\section{ABSTRACT \\ Effects of intake of different hydrating solutions on the hydra- tion levels and heart rate during a swimming exercise}

This study aimed to identify the hydration levels and interference in the heart rate as a result of adopting different hydration procedures during a swimming exercise with intervals. A total of 15 male athletes with age $18-26$ years ( $20.7 \pm 3.8$ years) was submitted to the following procedures: a) no type of hydration, b) hydration with placebo, and c) hydration with Gatorade ${ }^{\circledR}$. Each treatment corresponded to a total distance of 4150 meters divided in 250 meters of warm-up; $1 \times 400$ meters at maximum velocity; $1 \times 100$ meters in recovery; $10 \times 250$ meters at $85-90 \%$ of maximum velocity for this distance, with intervals of 50 meters in recovery and 400 meters at maximum velocity. $\mathrm{H}$ eart rate, body weight before and after each test, amount of liquid consumed during exercise and urine produced were measured to establish the hydration levels. No statistically significant difference $(p>0.05)$ was found on the time effect and between the groups in any of the two parameters analyzed. It can be concluded that the hydration procedures adopted did not influence the heart rate and hydration level responses during the experimental model developed.

Key-words: hydration, dehydration, swimming, heart rate 


\section{INTRODUÇÃO}

Nos treinamentos e provas de longa duração a hidratação deve ser constantemente realizada visando a manutenção da homeostase hídrica(23). Um procedimento de hidratação inadequado, ou mesmo a sua ausência, promoverá o aparecimento de um quadro de desidratação, podendo provocar alterações cardiovasculares e no equilíbrio hidroeletrolítico(14).

Observa-se que a maioria das pesquisas envolvendo hidratação e eventos de longa duração empregam o modelo de exercício da corrida ou do ciclismo.

Porém, os trabal hos que aplicam o modelo de exercício de natação relacionado com hidratação são escassos ou apenas são estudados quando ocorre a reprodução de um triathlon, como foi o caso das pesquisas desenvolvidas por Jeukendrup ${ }^{11}$ e Millard Stafford e colaboradores(19).

A natação, no entanto, apresenta condições especiais que modifica a relação da termogênese corporal, uma vez que o contacto do corpo com a água facilita a perda de calor e melhora a termogênese ${ }^{(17)}$. Outro factor diferenciador relaciona-se com o contacto da boca com a água durante todo o período de treinamento, o que estimula os receptores nervosos localizados na região orofaríngea, actuando assim como se o atleta estivesse continuamente se hidratando(15). Este tipo de estimulação nervosa faz com que o nadador não sinta sede, podendo provocar em muitas ocasiões uma ausência total de hidratação ao longo do treinamento.

A frequência cardíaca representa um importante parâmetro de controle do treinamento. Na natação a resposta da frequência cardíaca é diferente se comparada a um exercício como a corrida ou o ciclismo, pois o efeito imersão produz uma maior resposta bradicárdica(12). Considerando que a frequência cardíaca também sofre influência da desidratação, torna-se interessante investigar como será a resposta cronotrópica com diferentes acções de hidratação propostas ao longo de um treinamento de natação intervalado.

Tem-se assim como objectivo investigar os efeitos da ingestão de diferentes soluções hidratantes nos níveis de hidratação e na frequência cardíaca durante um exercício de natação intervalado

\section{MATERIAL E MÉTODOS}

Este estudo foi aprovado pelo Comitê de Ética em Pesquisa com Seres Humanos da Universidade Federal de Viçosa, de acordo com a resolução do Consel ho Nacional de Saúde nำ196/ 96, em consonância com as propostas das Diretrizes Éticas Internacionais para Pesquisas Biomédicas Envolvendo Seres Humanos (CIOMS/ OMS 1982 e 1993) e a declaração de Helsínquia (1989). Os voluntários receberam esclarecimentos detalhados sobre os procedimentos que seriam utilizados na colecta de dados e, em seguida, assinaram um termo de consentimento livre e esclarecido.

\section{Amostra}

Partici param na investigação 15 atletas do sexo masculino, pertencentes à Associação Atlética Acadêmica da Universidade Federal de Viçosa - Viçosa/MG e Minas Tênis Clube de Belo Horizonte/MG, com faixa etária compreendida entre 18 e 26 anos $(20,7 \pm 3,8$ anos). Todos os avaliados treinavam regularmente no mínimo 4 vezes por semana, possuíam total domínio das técnicas do nado e participavam regularmente de competições. As características antropométricas básicas dos aval iados foram de $180 \pm 7,3 \mathrm{~cm}$ para a estatura, $11 \pm 3,1 \%$ para o percentual de gordura corporal, sendo o peso corporal descrito na Tabela 2.

Procedimentos experimentais

Foi solicitado aos avaliados para que se abstivessem do treinamento por um período de 48 horas antes de cada uma das situações experimentais, visando não interferir na capacidade de armazenamento de glicogénio muscular e hepático. Solicitou-se ainda que mantivessem seus hábitos alimentares, inclusive os mesmos horários de refeição, durante o período de colecta de dados e que procurassem ter uma boa noite de sono.

\section{Desenho experimental}

Todos os avaliados foram submetidos no período da manhã ( 7:00 às 10:00 h) a quatro testes padronizados, diferenciados nas condições de hidratação, durante um período de tempo máximo de um mês de avaliação, dividido em quatro etapas: 1a Etapa: determinação da velocidade máxima em 
uma prova de natação estilo livre para uma distância total de 250 metros.

2a Etapa: exercício de natação intervalado, totalizando 4150 metros, e sem hidratação.

3a Etapa: exercício de natação intervalado, totalizando 4150 metros, e hidratação programada com solução carboidratada.

4a Etapa: exercício de natação intervalado, totalizando 4150 metros, e hidratação programada com solução placebo.

As quatro etapas foram realizadas no Departamento de Educação Física da UFV (Viçosa, Minas Gerais) e Minas Tênis Clube 2 (Belo Horizonte, Minas Gerias).

Foi planejado um desenho experimental, tendo como principal objectivo comparar as condições entre as situações de hidratação propostas. Para isto, foi adoptado um desenho cruzado e balanceado (crossover), de maneira que cada grupo de três avaliados, nas três ultimas etapas, iniciavam o procedimento de hidratação com uma acção distinta, caracterizando assim um desenho experimental denominado de quadrado latino(5).

Desenvolvimento do experimento

A massa corporal dos avaliados foi determinada com a utilização de uma balança digital Soehnl $e^{\circledR}$

(Espanha) com precisão de 100 gramas, estando o avaliado apenas de sunga.

O percentual de gordura foi estimado utilizando o método de três dobras cutâneas, sendo elas tríceps, tórax e subescapular, e posteriormente empregou-se a equação de Jackson \& Pollock(10). Para o registo das dobras cutâneas foi empregado o compasso científico Cescorf ${ }^{\circledR}$ (Brasil) com precisão de $1 \mathrm{~mm}$. Os procedimentos metodológi cos de mensuração de peso e estatura seguiram as orientações propostas por Marins e Giannichi(16).

\section{Procedimento protocolizado}

Na primeira etapa de testagem foi realizada a determinação da velocidade máxima em uma prova de natação estilo livre, para uma distância total de 250 metros. Nas três últimas etapas empregou-se o seguinte procedimento:

a) Aquecimento: 250 metros em ritmo livre, seleccionado pelo nadador. b) Primeira série de velocidade máxima em uma prova de 400 metros.

c) Recuperação activa de 100 metros em ritmo livre

d) Repetição de um total de dez séries de 250

metros a uma velocidade entre $85-90 \%$ da velocidade máxima da prova de 250 metros, seguida de intervalos activos de 50 metros.

e) Segunda série de velocidade máxima para uma prova de 400 metros.

Procedimentos de hidratação

Como o estudo era de característica duplo cego, as soluções de hidratação eram codificadas com numeração 148 e 226, sendo posteriormente identificadas como G atorade ${ }^{\circledR}$ e solução placebo, respectivamente. Antes de iniciar os protocolos, os atletas ingeriam água na proporção de $3 \mathrm{ml} / \mathrm{kg}$ de peso corporal. Durante o experimento também foi oferecido a mesma quantidade de líquido em 3 ocasiões. A Tabela 1 apresenta um resumo dos procedimentos metodológicos desenvolvidos no presente estudo.

Tabela 1. Ação metodológica de hidratação do estudo.

\begin{tabular}{|c|c|c|c|c|c|c|c|c|c|}
\hline Repc & Aqu. & $1 \stackrel{\mathrm{aS}}{ }$ & & $\begin{array}{l}\text { S1 S2 } \\
\text { S3 S4 }\end{array}$ & & S5* & $\begin{array}{c}\text { S6 S7 } \\
\text { S8 }\end{array}$ & $\begin{array}{l}\text { S9 } \\
\text { S10 }\end{array}$ & 2as \\
\hline Hid & $\underset{m}{250}$ & $\begin{array}{c}400 \\
m\end{array}$ & Hid & $\begin{array}{c}250 \\
m\end{array}$ & Hid & $\begin{array}{c}250 \\
m\end{array}$ & $\begin{array}{c}250 \\
m\end{array}$ & $\begin{array}{c}\text { Hid } 250 \\
\text { m }\end{array}$ & $\begin{array}{c}400 \\
\mathrm{~m}\end{array}$ \\
\hline
\end{tabular}

Procedimentos para mensuração do nível de hidratação e frequência cardíaca

Nível de hidratação

O nível de hidratação foi considerado pelo comportamento do peso corporal dos avaliados. Para isto, o peso corporal foi mensurado antes e depois de cada prova, utilizando a balança referida anteriormente. Para sua mensuração, o avaliado era pesado apenas de sunga, sendo que após o treino o nadador secava seu corpo com uma toal ha antes de ser pesado. Para a análise da perda hídrica absoluta, foi considerado peso mensurado no final do teste menos peso inicial, mais quantidade de líquido consumido durante o experimento. Já a perda hídrica relativa foi cal culada pela diferença registada na bal ança entre o peso final e o inicial, desconsiderando o líquido ingerido. 
Tabela 2. Resultado do peso corporal (PC) em Kg e suas variações antes e depois das testagens, adotando diferentes procedimentos de hidratação.

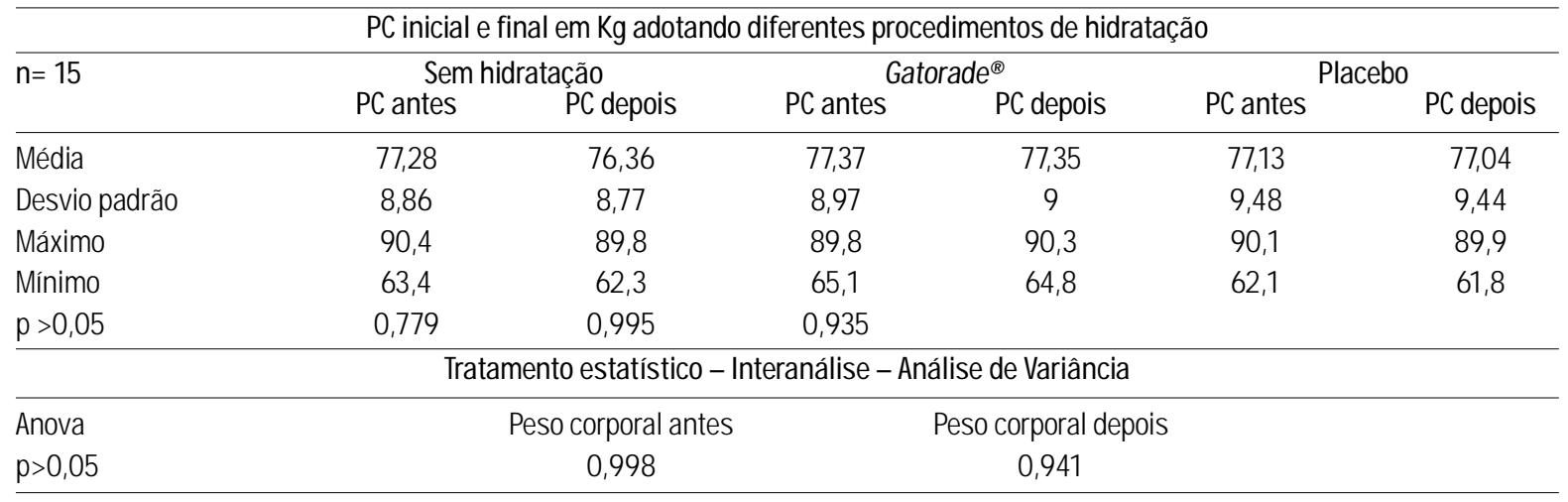

Tabela 3. Perda hídrica absoluta e relativa com adopção dos diferentes tratamentos

\begin{tabular}{lccccccccc}
\hline \multicolumn{10}{c}{ Perda hídrica relativa (PR), perda hídrica absoluta (PA) em Kg e \%PA nos Diferentes tratamentos } \\
\hline $\mathrm{n}=15$ & \multicolumn{3}{c}{ Sem hidratação } & \multicolumn{3}{c}{ Gatorade ${ }^{\circledR}$} & \multicolumn{3}{c}{ Placebo } \\
& $\mathrm{PA}(\mathrm{Kg})$ & $\mathrm{PR}(\mathrm{kg})$ & $\% \mathrm{PA}$ & $\mathrm{PA}(\mathrm{kg})$ & $\mathrm{PR}(\mathrm{kg})$ & $\% \mathrm{PR}$ & $\mathrm{PA}(\mathrm{kg})$ & $\mathrm{PR}(\mathrm{kg})$ & $\% \mathrm{PR}$ \\
\hline Média & $-0,91$ & $-0,91$ & 1,18 & $-0,95$ & $-0,02$ & 0,025 & $-0,99$ & 0,09 & 0,116 \\
Desvio padrão & 0,36 & 0,36 & 0,47 & 0,32 & 0,30 & 0,38 & 0,26 & 0,23 & 0,295 \\
Máximo & $-1,6$ & $-1,6$ & 1,89 & $-1,47$ & $-0,4$ & 0,47 & $-1,55$ & $-0,5$ & 0,57 \\
Mínimo & $-0,4$ & $-0,4$ & 0,6 & $-0,53$ & $+0,5$ & $\mathrm{NC}$ & $-0,58$ & $+0,4$ & $\mathrm{NC}$ \\
\hline
\end{tabular}

NC: Houve ganho - hiperhidratação

Tabela 4. Cota de produção de suor em $\mathrm{ml} /$ minuto empregando os diferentes tratamentos

\begin{tabular}{lccc}
\hline \multicolumn{4}{c}{ Cota de produção de suor em ml/minuto } \\
\hline $\mathrm{n}=15$ & Sem hidratação & Gatorade ${ }^{\circ}$ & Placebo \\
\hline Média & 11,75 & 11,97 & 12,5 \\
Desvio padrão & 4,71 & 3,86 & 3,38 \\
Máximo & 20,6 & 18,44 & 19,97 \\
Mínimo & 7,24 & 7,36 & 7,33 \\
\hline \multicolumn{4}{c}{ Tratamento estatístico - Interanálise - Análise de Variância } \\
\hline p $>0,05$ & \multicolumn{4}{c}{0,872} \\
\hline
\end{tabular}


Tabela 5. Frequência cardíaca máxima registada nas séries de 250 metros

\begin{tabular}{|c|c|c|c|c|c|c|c|c|c|c|c|c|c|c|c|}
\hline \multirow[t]{2}{*}{$\mathrm{n}=15$} & \multicolumn{4}{|c|}{ Sem ingestão de líquido } & \multirow[t]{2}{*}{ Anova } & \multicolumn{4}{|c|}{ Gatorade ${ }^{\circledR}$} & \multirow[t]{2}{*}{ Anova } & \multicolumn{4}{|c|}{ Placebo } & \multirow[t]{2}{*}{ Anova } \\
\hline & Média & DP & Máx & Min & & Média & $\mathrm{DP}$ & Máx & Min & & Média & $\mathrm{DP}$ & Máx & Min & \\
\hline Série1 & 172 & 10,5 & 189 & 150 & \multirow[t]{10}{*}{0,998} & 171,9 & 9,84 & 195 & 157 & \multirow[t]{10}{*}{0,994} & 168,2 & 8,05 & 183 & 157 & \multirow[t]{10}{*}{0,960} \\
\hline Série2 & 173 & 9,88 & 189 & 155 & & 172,7 & 8,87 & 192 & 159 & & 170,1 & 8,54 & 181 & 148 & \\
\hline Série3 & 172 & 10,1 & 186 & 150 & & 174 & 8,85 & 192 & 159 & & 172,3 & 7,15 & 182 & 161 & \\
\hline Série4 & 173 & 9,45 & 188 & 152 & & 173,5 & 8,82 & 190 & 161 & & 171,8 & 7,85 & 183 & 157 & \\
\hline Série5 & 174 & 7,98 & 188 & 161 & & 173,4 & 9,02 & 192 & 162 & & 171,5 & 8,72 & 185 & 155 & \\
\hline Série6 & 171 & 8,84 & 189 & 158 & & 170,9 & 9,95 & 190 & 159 & & 168,3 & 9,39 & 182 & 151 & \\
\hline Série7 & 172 & 8,83 & 188 & 157 & & 172,4 & 8,10 & 187 & 162 & & 169,4 & 10,29 & 186 & 152 & \\
\hline Série8 & 173 & 8,43 & 187 & 158 & & 174,1 & 11,16 & 201 & 159 & & 169,7 & 12,08 & 188 & 142 & \\
\hline Série9 & 172 & 8,29 & 188 & 158 & & 172,1 & 7,67 & 183 & 158 & & 169,4 & 11,77 & 187 & 143 & \\
\hline Série10 & 173 & 8,52 & 187 & 161 & & 171,6 & 9,62 & 189 & 158 & & 168,7 & 11,94 & 187 & 140 & \\
\hline Anova & \multicolumn{15}{|c|}{ Tratamento estatístico - Interanálise - Análise de variância } \\
\hline \multirow[t]{2}{*}{$p>0,05$} & \multirow{2}{*}{\multicolumn{2}{|c|}{$\begin{array}{l}\text { Série1 } \\
0.482\end{array}$}} & \multicolumn{2}{|c|}{ Série2 } & Série3 & Série4 & \multirow{2}{*}{\multicolumn{2}{|c|}{ Série5 }} & Série & \multirow{2}{*}{\multicolumn{2}{|c|}{$\begin{array}{c}\text { Série7 } \\
0,6\end{array}$}} & Série8 & \multirow{2}{*}{\multicolumn{2}{|c|}{$\begin{array}{c}\text { Série9 } \\
0,672\end{array}$}} & Série10 \\
\hline & & & & & 0,829 & 0,838 & & & 0,69 & & & 0,511 & & & 0,53 \\
\hline
\end{tabular}

Tabela 6. Frequência cardíaca máxima média registada nas duas séries de 400 metros empregando diferentes tratamentos.

\begin{tabular}{|c|c|c|c|c|c|c|c|c|c|}
\hline $\mathrm{N}=15$ & & Série $1 \mathrm{~d}$ & metros & & & érie 20 & metr & & \\
\hline & Média & DP & Máx & Min & Média & DP & Máx & Min & Teste $\mathrm{t}$ \\
\hline Sem líquido & 174,9 & 11,19 & 192 & 153 & 174,7 & 12,62 & 198 & 143 & 0,976 \\
\hline Gatorade ${ }^{\circledR}$ & 177,2 & 12,39 & 205 & 155 & 177,5 & 10,9 & 198 & 160 & 0,938 \\
\hline Placebo & 171,3 & 12,48 & 187 & 138 & 176,5 & 12,71 & 193 & 146 & 0,274 \\
\hline
\end{tabular}

Portanto, com base no resultado final da bal ança e no líquido consumido, foi possível estabel ecer a perda hídrica absoluta, a perda hídrica relativa, a taxa de produção de suor por minuto e o percentual de desidratação.

A frequência cardíaca foi registada em repouso e durante cada intervalo de 5 segundos, durante todo o transcurso do experimento, com as diferentes situações de hidratação, utilizando o monitor de frequência cardíaca da marca Polar ${ }^{\circledR}$ modelo A curex Plus $^{\circledR}$. Este equipamento possui Interface Plus ${ }^{\mathrm{TM}}$ Training A dvisorTM com software for Windows ${ }^{\circledR}$. A análise estatística da frequência cardíaca tomou como referência o val or da frequência mais al ta obtida no final de cada série nadada.

Fatores intervenientes

Durante o transcurso das etapas, vários factores poderiam intervir de maneira directa ou indirecta, provocando alterações dos resultados. Para minimizar a interferência destes elementos externos à investigação foram mensurados al guns factores como, temperatura da água da piscina que se manteve durante o período de testagem em média com $26 \pm 2$ 으 C; horário de real ização dos testes, sempre no período da manhã; nível de hidratação e quantidade de líquido hidratante, adoptando-se a quantidade de $3 \mathrm{ml} / \mathrm{kg}$ de peso corporal.

Tratamento estatístico

O sistema informático Primer ${ }^{\circledR}$ foi utilizado para realização das análises estatísticas, sendo que para avaliação da homogeneidade das distribuições utilizouse o teste de Kolmogorov-Smirnov. A pós esta avaliação em uma primeira etapa, empregou-se uma análise estatística descritiva e na etapa seguinte, utilizou-se 
uma estatística inferencial com o teste de ANOVA "O ne Way" para medidas repetidas, a fim de identificar as diferenças entre os procedimentos de hidratação, e cota de produção de suor, correspondendo, assim, a uma análise intra-grupo. $O$ teste t pareado foi utilizado para verificar diferença no peso antes e depois de cada procedimento (análise inter-grupo). Adoptou-se o nível de significância $p<0,05$ para considerar válida a hi pótese estatística.

\section{RESULTADOS}

A tabela 2 apresenta o peso corporal dos avaliados, antes e após cada tratamento, enquanto a Tabela 3 e 4 referem-se respectivamente ao percentual de perda hídrica absoluta e relativa dos nadadores e sua cota de produção de suor.

Observa-se que embora tenha ocorrido redução do peso corporal em todos os tratamentos esta redução não foi estatisticamente significativa $(p>0,05), 0$ mesmo ocorrendo com a cota de produção de suor. Avaliando os valores de frequência cardíaca entre as séries de 250 e 400 metros apresentados respectivamente nas Tabelas 5 e 6, também não se verificou diferença estatisticamente significativa.

\section{DISCUSSÃO}

Nível de hidratação

Observou-se uma diminuição média entre o peso inicial e final dos atletas adoptando os diferentes procedimentos, com redução de 0,913 $\pm 0,36 \mathrm{~kg}$ para a situação sem hidratação, o que representa uma desidratação de $1,18 \pm 0,47 \%$. Para as situações ingestão de Gatorade ${ }^{\circledR}$ e placebo, a perda hídrica foi de $0,02 \pm 0,3 \mathrm{~kg}$ e 0,093 $\pm 0,23 \mathrm{~kg}$ respectivamente. Estas diferenças não foram consideradas estatisticamente significativa nos três procedimentos adoptados, mesmo incluindo a situação não hidratando. Estes resultados são totalmente contraditórios, quando comparados durante modelos semelhantes em ambientes terrestres de corrida, onde a não hidratação é responsável por uma perda hídrica aguda(15, 17). Uma das justificativas para a ausência de diferenças entre as condições de hidratação, frente à não hidratação na natação é a termogênese facilitada no meio líquido, de forma que a produção de sudorese é minimizada, sendo a perda de calor obtida através da condução e convecção.
Apesar de ocorrer uma redução de peso corporal em condições de ausência de hidratação de $910 \pm 360 \mathrm{~g} \mathrm{e}$ praticamente não haver sido registado perda de peso nas duas condições onde manteve-se o nadador hidratado constantemente estes resul tados estatisticamente não foram considerados como significativos no comportamento do peso corporal ao longo do treinamento de natação evidenciado neste estudo. Isto torna claro que a desidratação como elemento redutor de performance assume um papel secundário, se comparado ao que ocorre com o exercício de corrida e cicl ismo.

É evidente que não se trata de recomendar a não hidratação, porém adaptar as recomendações do $\operatorname{ACSM}^{(1)}$. É interessante destacar que o procedimento nutricional durante um treino de natação possa ter como foco principal a reposição energética, seja ela sobre a forma de barras energéticas, gel ou bebidas com maior concentração de carboidratos, oferecidos em intervalos superiores aos habituais 15 minutos(1). A reposição energética virá neste caso minimizar o risco de um quadro de hipoglicemia. O percentual de desidratação observado pela perda de peso dos atletas deste estudo quando não ingeriram líquidos foi em média de $1,18 \pm 0,47 \%$, estando em conformidade com o estudo de Krug(13) que encontrou um percentual de desidratação em nadadores de $1,5 \%$ real izando um treinamento de natação com distância total de 4900 metros, ou seja, 750 metros a mais que o presente estudo. Estes val ores de desidratação são inferiores ao valor crítico de $2 \%$, que é suficiente para redução na performance(24). Os resultados do presente estudo e do estudo de Krug(13) demonstram que actividade física sem ingestão de líquido leva a uma desidratação, fato comprovado pela perda de peso corporal. No entanto, observa-se que a redução de peso na natação é menor que em outros esportes de longa duração como, por exemplo, a maratona onde as perdas hídricas podem al cançar quatro litros(4).

Contrariamente aos dados apresentados até aqui, Diprampero et al.(9) observaram perda de peso corporal em nadadores entre 2 e $3 \mathrm{~kg}$, durante sessões de treinamento, comprovando que mesmo com a termogênese facilitada pelo meio líquido a desidratação pode ser alta dependendo da temperatura da água e de características individuais dos avaliados. 
A ausência de diferença entre o grau de perda hídrica quando comparadas 2 formas de hidratação ( $G$ atorade ${ }^{\circledR}$ versus placebo) não chega a surpreender, já que outros estudos também não obtiveram diferença durante exercícios de corrida(25) e ciclismo(2, 14).

A Figura 1 demonstra que, as maiores perdas de peso absolutas encontradas neste estudo, ocorreram com os voluntários 9 e 15, que perderam respectivamente 1,6 kg não hidratando e 1,55 kg hidratando com $\mathrm{G}$ atorade ${ }^{\circledR}$. Estes atletas em particular devem manter um maior nível de atenção para a hidratação, já que a desidratação influencia a performance, e eles possuem uma taxa de sudorese al ta para a natação (20,6 e 19,97 ml/minuto, respectivamente).

Por outro lado, a menor diminuição de peso absoluto ocorreu com o avaliado 11 perdendo apenas 0,4 $\mathrm{kg}$ quando não se hidratou, produzindo uma taxa de sudorese de 5,24 ml/min. Esta diferença na perda de peso entre os atletas pode ser explicada por factores individuais. A Figura 1 apresenta de forma clara como que a resposta de produção de suor é especificamente individual, porém reprodutível no mesmo sujeito.

Considerando a resposta individual de produção de suor, é recomendável que cada nadador controle seu peso corporal antes e depois do treinamento, facilitando o planejamento da curva de recuperação dos fluidos corporais, que segundo Maughan e Shirrefs(18) deverá corresponder a $150 \%$ do peso perdido, ou seja, caso a diferença seja de $500 \mathrm{~g}$ o nadador deverá consumir $750 \mathrm{~g}$ ou o equivalente a 750 $\mathrm{ml}$ de líquido após o treino.

Outro fato relevante observado é que ao ingerir solução carboidratada ou placebo al guns atletas tiveram um ganho de peso como, por exemplo, o avaliado 7 que ganhou 500 gramas ao hidratar com $\mathrm{G}$ atorade ${ }^{\circledR}$ e o avaliado 14 que ganhou 400 gramas ao hidratar com placebo e Gatorade $\AA$ (Figura 2). No caso destes dois atletas pode-se inferir que em treinamentos longos (de 4 horas de duração ou mais) eles poderiam ter um risco maior de hiperhidratação, caso mantivessem a mesma cota de hidratação proposta neste estudo. É importante destacar que durante o período de testagem não se registou perda hídrica por via urinária em nenhum voluntário.

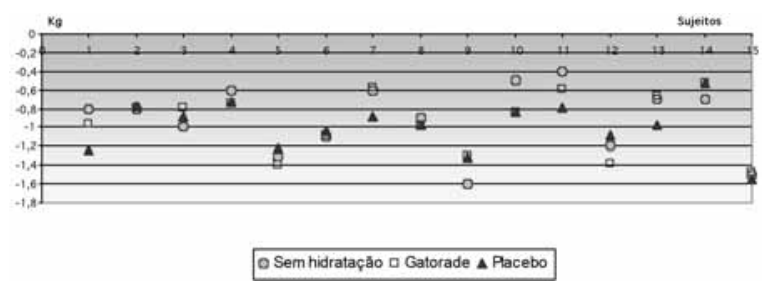

Figura 1. Perda absoluta de peso dos 15 avaliados

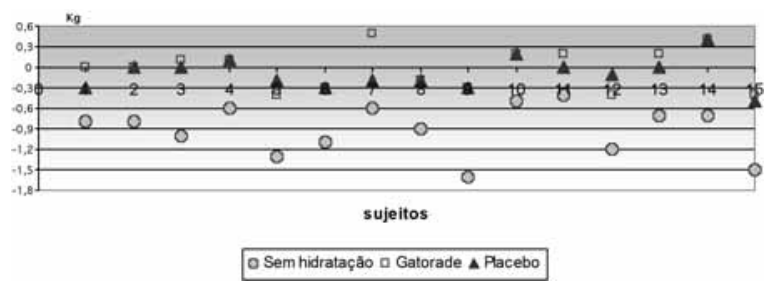

Figura 2. Perda relativa de peso dos 15 avaliados

Frequência cardíaca

Neste estudo não se verificou diferença estatística no comportamento da frequência cardíaca inter-grupo, comparando as três diferentes condições de hidratação, e intra-grupo comparando a FC ao longo de cada exercício entre as dez séries. Este resultado pode ser justificado devido ao baixo nível de desidratação médio apresentado pelos atletas

$(1,18 \% \pm 0,47 \%$ de perda de peso). Este grau de desidratação não foi suficiente para promover um incremento na frequência cardíaca dos atletas quando estes não ingeriam líquidos. Estes dados estão em conformidade com os dados de Krug(13) que também não observou diferença estatisticamente significativa na frequência cardíaca de nadadores, com níveis de desidratação de aproximadamente 1,5\%.

Em situações de exercício com e sem hidratação, observa-se que não hidratando ocorre um aumento na frequência(21), sendo que esta resposta da elevação da frequência cardíaca no estado desidratado provavelmente é decorrente da redução do volume sanguíneo(14, 7, 22). No entanto, no presente estudo as oscilações observadas de FC média durante as 10 séries de 250 metros, não foram suficientes para o teste de ANOVA detectar diferenças significativas, indicando assim uma resposta constante do coração, independentemente da série. Comportamento semeIhante foi obtido entre a FC média da $1^{\circ}$ e 2 으 séries 
de 400 metros, ao não ser identificada diferenças significativas através do testet pareado, quando analisada de forma independente em cada uma das três situações estudadas.

Estes resultados surpreendem quando se avaliam as duas condições de hidratação frente à não hidratação. Em trabal hos com este tipo de acção metodológica, porém realizadas através do exercício de corrida e ciclismo os resultados apontam para uma elevação desta cota da frequência cardíaca quando da não hidratação. Observa-se claramente na natação uma menor elevação da frequência cardíaca quando da não hidratação frente ao hidratado em ambiente terrestre. Os possíveis factores influenciadores para esta resposta atípica seriam: a) termogênese facilitada; b) menor índice de desidratação; c) posição do corpo em exercício.

O aumento contínuo da frequência cardíaca ao longo do exercício, conhecido como cardiovascular "drift" é decorrente de quadros de desidratação, o que promove uma redução do volume plasmático, consequentemente do volume sistólico. Visando equilibrar o débito cardíaco, tem-se como resposta adaptativa o aumento da frequência cardíaca(14, 26). Neste estudo, os níveis de desidratação produzidos nas três condições avaliadas não foram suficientes para influenciar o sistema cardiovascular e apresentar este fenómeno. A ausência de diferença significativa na resposta da frequência cardíaca entre os dois procedimentos de hidratação não surpreendem, tendo em vista que concordam com outros trabal hos que comparam a interferência na frequência cardíaca ao se adoptar solução carboidratada versus água, de maneira que a frequência cardíaca não varia em função dos diferentes procedimentos de hidratação utilizados $(6,14,20)$. Entretanto, outros estudos demonstram que há variação quando são comparados diferentes bebidas $(3,8)$. No entanto, provavelmente esta variação está mais relacionada à quantidade de líquido oferecido, não havendo razões teóricas que justifique a diferença entre as bebidas, a não ser quando existe uma diferença no tempo de esvaziamento gástrico entre os líquidos que possa interferir na hidratação.

\section{CONCLUSÕES}

Os diferentes tipos de hidratação empregados neste estudo não modificaram significativamente a respos- ta do nível de hidratação e da frequência cardíaca dos atletas.

Características individuais de variação do peso corporal podem aumentar a perda hídrica do nadador, minimizando o efeito mais termogênico do meio líquido, impondo uma desidratação superior a $2 \%$ do peso corporal.

Os níveis de desidratação obtidos não foram suficientes para promover o aumento contínuo da frequência cardíaca ao longo do exercício.

\section{AGRADECIMENTOS}

Ao PIBIC/CNPq pela concessão da bolsa de iniciação científica, ao Gatorade Sport Science Institute pelo financiamento do projecto e aos clubes e atletas participantes da pesquisa.

\section{CORRESPONDÊNCIA}

\section{Fabrícia Geralda Ferreira}

Universidade Federal de Viçosa

Departamento de Educação Física Laboratório de Performance Humana Viçosa, MG, Brasil - CEP.: 36571-000 Tel.: (55) 3138992249 - (55) 38-91062434 E-mail: fafege@yahoo.com.br 


\section{REFERÊNCIAS}

1. American College Sports Medicine (1996). Position Stand. Exercise and fluid Replacement. M ed Sci Sports Exerc 28 (1): i - vii.

2. Almeida G, Cocate $P$, Carvalho $M$, Marins N, Marins J (2004). Perda de peso absoluta (KG e \%) adotando dois procedimentos de hidratação durante uma prova de ciclismo. Revista M ineira Educação Física 12 (2): 562.

3. Angus D, Hargreaves M, Dancey J, Febraio M (2000). Effect of carbohydrate or carbohydrate plus medium- chain triaglyceride ingestion on cycling time trial performance. J A ppl Physiol 88:113-119.

4. Batlle J (1992). Alimentación del deportista: aplicación prática en el deport. Raciones de entrenamiento, competicón y recuperación. A porte hidro-mineral. A punts 25 (5): 271-282.

5. Bravo R (1996). Tesis doctoral y trabajos de investigación científica. Madrid: Paraninfo.

6. Brisswater J, Hausswirth C, Vercruyssen F, Collardeau M, Vallier J, Lepers R, Goubault C (2000). Carboydrate ingestion does nor influence the change in energy cost during a 2-h run in well- trained triathletes. Eur J A ppl Physiol 81:108 - 113.

7. Coyle E, Montain S (1993). Thermal and cardiovascular responses to fluid during exercise. M ed Sci Sports Exerc 6: 179-224.

8. Davis J, Welsh R, DE - Volve K, Alderson N (1999). Effects of branched - chain amino acids and carbohydrate on fadigue during intermittent high - intensity running. Int J Sports M ed 20: 309 - 314.

9. Diprampero P, Pendergast D, Wilson D, Rennie D (1978). Blood lactic acid concentrations in high velocity swimming. In: Eriksson B, Furberg B (ed.). Swimming M ed IV : 249-261.

10. Jackson A, Pollock M (1985). Assessment of body composition Phys. Sport M ed 13: 76 - 90.

11. Jeukendrup A, Jentjens R, Moseley L (2005). Nutritional considerations in triathlon. Sport M ed 35 (2): 163-81

12. Kruel L, Peyré-Tartaruga L, Dias A, Silva R, Picanço P, Rangel A (2002). Freqüência cardíaca durante imersão no meio aquático. Fit. \& Perform 1(6): 46-51.

13. Krug M (1997). A nálise de variáveis fisiológicas durante uma sessão de treinamento com e sem ingestão de água em nadadores masculinos. Dissertação (Mestrado em Ciência e Movimento) - Universidade Federal de Santa Maria.

14. Marins J (2000). Estudio comparativo de diferentes procedimientos de hidratación durante un ejercicio de larga duración. Tese (Doutorado em Biologis) Universidad de Murcia, Espanha.

15. Marins J (1993). Influencia da ingestão de Gatorade por atletas no desempenho físico em provas eminentemente aeróbicas. Dissertação (Mestrado em Educação Física - Escola de Educação Física e Desportos,) Universidade Federal do Rio de Janeiro, Rio de Janeiro.

16. Marins J, Giannichi R (2003). Avaliação e Prescrição de A tividade Física: Guia Prático. 3. ed. Rio de Janeiro: Shape.

17. Mcardle W, Katch F, Katch V (2003). Fisiologia do Exercício: Energia, N utrição e D esempenho H umano. 6a ed. Rio de Janeiro: Guanabara Koogan.

18. Maughan R, Shirrefs S (1998). Dehydration, rehydration and exercise in the heat - concluding remarks. Int J Sports M ed 19: 167 - 168.
19. Millard-Stafford M, Sparling $P$, Rosskopf $L$, Hinson B, Diarlo L (1990). Carbohydrate-electrolyte replacement during a simulated triathlon in the heat. M ed Sci Sports Exerc 22 (5): 621-628.

20. Peters $\mathrm{H}$, Wiersma W, Akkermans L, Bol E, Kraajenhagen R, Mosterd W (2000). Gastrointestinal mucosal integrity after prolonged exercise with fluid suplementation. M ed Sci Sports Exerc 32 (1): 134-142.

21. Saat M, Tochihara $Y$, Hashiguchi N, Sirisinghe R, Fujita $M$, Chou C (2005). Effects of Exercise in the Heat on Thermoregulation of Japanese and Malaysian Males. J Physiol A nthropol A ppl Human Sci 24(4): 267-275.

22. Saltin B (1964). Aerobic and anaerobic work capacity after dehydration. J A ppl Physiol 19 (6):1114-1118.

23. Sawka M, Cheuvront S, Carter R (2005). Human Water Needs. Nutr Rev 63 (6): S30-S39.

24. Sawka M, Latzka W, Mattot R, Montain S (1998). Hydration Effects on Temperature Regulation. Int J Sports M ed 19: S108-S110.

25. Wilmore J, Morton A, Gilbey H, Wood R (1998). Role of taste preference on fluid inteka during and after 90 min of running at $60 \%$ of Vo2máx in the heat. M ed Sci Sports Exerc 4: 587 - 595 .

26. Wingo J, Lafrenz A, Ganio M, Edwards G, Cureton K (2005). Cardiovascular drift is related to reduced maximal oxygen uptake during heat stress. M ed Sci Sports Exerc 37 (2):248-255 